\title{
MOTION AND HOMOGENIZATION OF VORTICES IN ANISOTROPIC TYPE II SUPERCONDUCTORS*
}

\author{
S. J. CHAPMAN ${ }^{\dagger}$ AND G. RICHARDSON ${ }^{\dagger}$
}

\begin{abstract}
The motion of vortices in an anisotropic superconductor is considered. For a system of well-separated vortices, each vortex is found to obey a law of motion analogous to the local induction approximation, in which velocity of the vortex depends upon the local curvature and orientation. A system of closely packed vortices is then considered, and a mean field model is formulated in which the individual vortex lines are replaced by a vortex density.
\end{abstract}

Key words. superconductivity, Ginzburg-Landau equations, mean-field, anisotropic, hightemperature

AMS subject classification. $82 \mathrm{D} 55$

PII. S0036139995282682

1. Introduction. Type II superconductors are characterized by the appearance of line singularities known as normal filaments or vortices. These singularities carry a magnetic field, and associated with this magnetic field is a circulating superconducting current (hence the term vortex). These structures are immensely important in technological applications, since any superconductor which is in the presence of a high magnetic field will contain millions of vortices. In particular, it is important to understand how vortices move under the influence of an electric current, since movement causes dissipation, which leads to an electrical resistance.

Moreover, since there will be many millions of vortices in any reasonably sized sample, it is important to be able to formulate a mean-field, or averaged model, in which the individual vortices have been replaced by a vortex density, or vorticity.

Recently, in [6], [4] both the law of motion of vortices and a mean-field model have been formulated, using the Ginzburg-Landau theory of superconductivity [11], $[3],[8]$ as a starting point.

Many of the high-temperature superconducting materials, however, do not fit immediately into the Ginzburg-Landau framework. A typical characteristic of these materials is that of a layered structure, with superconducting planes being separated by regions of normal (nonsuperconducting) material. Thus, at the very least, these materials are highly anisotropic, with different characteristics across planes to those in plane.

Two models have been proposed to deal with such materials. The first is the Lawrence-Doniach model, in which the individual planes are modelled by the Ginzburg-Landau theory and coupled via Josephson tunnelling between the planes, leading to a system of differential-difference equations [15]. An alternative model is the anisotropic Ginzburg-Landau model, which is a variation of the Ginzburg-Landau theory which takes account of the anisotropy of the high- $T_{c}$ materials. It has been shown recently in [5] that in the limit of strong Josephson coupling, the Lawrence-

${ }^{*}$ Received by the editors March 8, 1995; accepted for publication (in revised form) October 23, 1996. The research of the first author was supported by a British Nuclear Electric Research fellowship. The research of the second author was supported by a Science and Engineering Research Council studentship.

http://www.siam.org/journals/siap/58-2/28268.html

†Mathematical Institute, Oxford University, 24-29 St. Giles', Oxford OX1 3LB, UK (chapman@ maths.ox.ac.uk). 
Doniach model approaches the anisotropic Ginzburg-Landau model as the separation between the planes tends to zero.

The aim of the present work is to extend the analysis in [6] and [4] to the case of an anisotropic superconductor. In section 2 we introduce the anisotropic time-dependent Ginzburg-Landau equations written down by Genkin and Mel'nikov [10]. In section 3 we perform an asymptotic analysis of a system of well-separated vortices and derive the law of motion as an anisotropic version of the local induction approximation. In section 4 we consider a system of densely packed vortices and formulate a mean-field model in which the law of motion of vortices is derived from an asymptotic analysis of each vortex core. In section 5 we consider some examples of vortex motion under the law derived in section 3. Finally, in section 6 we present our conclusions.

2. The anisotropic time-dependent Ginzburg-Landau model. The anisotropic time-dependent Ginzburg-Landau equations written down by Genkin and Mel'nikov [10] are

$$
\begin{array}{r}
\hbar \gamma \frac{\partial \psi}{\partial t}+2 i e \gamma \psi \Phi+b \psi|\psi|^{2}+a \psi+\sum_{k=1}^{3} \frac{1}{4 m_{k}}\left(i \hbar \frac{\partial}{\partial x_{k}}+2 e A_{k}\right)^{2} \psi=0 \\
\mathbf{j}=-\sum_{k=1}^{3} \sigma_{k}\left(\frac{\partial A_{k}}{\partial t}+\frac{\partial \Phi}{\partial x_{k}}\right) \mathbf{e}_{\mathbf{k}}-\frac{2 e^{2}}{m_{k}}\left(|\psi|^{2} A_{k}+\frac{i \hbar}{4 e}\left(\psi^{*} \frac{\partial \psi}{\partial x_{k}}-\psi \frac{\partial \psi^{*}}{\partial x_{k}}\right)\right) \mathbf{e}_{\mathbf{k}}
\end{array}
$$

Here, $\psi$ is the complex order parameter (such that $|\psi|=\sqrt{\frac{|a|}{b}}$ represents wholly superconducting material, $\psi=0$ represents wholly normal material $), \mathbf{A}=\left(A_{1}, A_{2}, A_{3}\right)$ is the real magnetic vector potential, $\Phi$ is the electric scalar potential, $\mathbf{j}$ is the electric current density, $e$ is the charge on an electron, $\hbar$ is Planck's constant, and $\sigma_{k}$ represents the conductivities of the ohmic component of the current in the principal directions; $\gamma, a, b$, and $m_{i}$ are phenomenological parameters: $\gamma$ is a relaxation parameter for the superconducting electrons, $a$ and $b$ occur as the coefficients in the expansion of the free energy in powers of $|\psi|^{2}$ (below the critical temperature $a<0, \quad b>0$ ), and the $m_{i}$ are the parameters which represent the anisotropy of the material. These equations must be coupled to the Maxwell equation

$$
\mathbf{j}=\nabla \wedge \mathbf{H}
$$

where $\mathbf{H}$ is the magnetic field, given by $\mu \mathbf{H}=\nabla \wedge \mathbf{A}$.

We nondimensionalize by introducing new variables as follows:

$$
\begin{array}{ccc}
\psi=\sqrt{\frac{|a|}{b}} \psi^{\prime}, & \mathbf{H}=|a| \sqrt{\frac{2}{b \mu}} \mathbf{H}^{\prime}, & \mathbf{A}=\frac{\sqrt{m|a|}}{e} \mathbf{A}^{\prime}, \\
\mathbf{x}=\frac{1}{e} \sqrt{\frac{m b}{2|a| \mu}} \mathbf{x}^{\prime}, & \sigma_{k}=\delta \sigma_{k}^{\prime}, & \Phi=\frac{\hbar|a|}{4 \gamma m} \sqrt{\frac{2 \mu}{b}} \Phi^{\prime}, \\
t=\frac{2 \gamma m^{2} b}{e^{2}|a| \mu \hbar} t^{\prime}, & m_{k}=m m_{k}^{\prime},
\end{array}
$$

where $m$ and $\delta$ are chosen so that $m_{1}^{\prime} m_{2}^{\prime} m_{3}^{\prime}=1$ and $\sigma_{1}^{\prime} \sigma_{2}^{\prime} \sigma_{3}^{\prime}=1$. These are the standard nondimensionalizations of the Ginzburg-Landau model; the lengthscale is the magnetic decay length, or penetration depth, and the scale for the magnetic field is the thermodynamic critical field. On dropping the primes, the nondimensional 
equations are

$$
\begin{aligned}
-\nabla \wedge(\nabla \wedge \mathbf{A})= & \sum_{k=1}^{3} \frac{\sigma_{k}}{\alpha}\left(\frac{\partial A_{k}}{\partial t}+\frac{\partial \Phi}{\partial x_{k}}\right) \mathbf{e}_{\mathbf{k}} \\
& +\sum_{k=1}^{3} \frac{1}{m_{k}}\left(\frac{i}{2 \kappa}\left(\psi^{*} \frac{\partial \psi}{\partial x_{k}}-\psi \frac{\partial \psi^{*}}{\partial x_{k}}\right)+|\psi|^{2} A_{k}\right) \mathbf{e}_{\mathbf{k}}, \\
\mathbf{H}= & \nabla \wedge \mathbf{A},
\end{aligned}
$$

where

$$
\kappa=\frac{m}{e \hbar} \sqrt{\frac{2 b}{\mu}}, \quad \alpha=\frac{4 \gamma m}{\hbar \delta \mu} .
$$

Equations (5)-(8) reduce to the standard nondimensional Ginzburg-Landau equations when $m_{i}^{\prime}=\sigma_{i}^{\prime}=1$ for $i=1,2,3$, where $\kappa$ is the Ginzburg-Landau parameter.

Equations (5)-(8) are gauge invariant in the sense that they are invariant under transformations of the form

$$
\mathbf{A} \rightarrow \mathbf{A}+\nabla w, \quad \Phi \rightarrow \Phi-\frac{\partial w}{\partial t}, \quad \psi \rightarrow \psi e^{i \kappa w} .
$$

We may take advantage of this invariance to write the equations in terms of real variables by introducing appropriately scaled gauge-invariant potentials

$$
\begin{gathered}
\mathbf{Q}=\kappa \mathbf{A}-\nabla \chi \\
\Theta=\kappa \Phi+\frac{\partial \chi}{\partial t},
\end{gathered}
$$

where $\psi=f e^{i \chi}$. We find

$$
\begin{array}{r}
\epsilon^{2} \frac{\partial f}{\partial t}+\sum_{k=1}^{3} \frac{\epsilon^{2}}{m_{k}}\left(f Q_{k}^{2}-\frac{\partial^{2} f}{\partial x_{k}^{2}}\right)-f+f^{3}=0, \\
f^{2} \Theta+\sum_{k=1}^{3} \frac{1}{m_{k}} \frac{\partial}{\partial x_{k}}\left(f^{2} Q_{k}\right)=0, \\
-\nabla \wedge(\nabla \wedge \mathbf{Q})=\sum_{k=1}^{3} \frac{\sigma_{k}}{\alpha}\left(\frac{\partial Q_{k}}{\partial t}+\frac{\partial \Theta}{\partial x_{k}}\right) \mathbf{e}_{\mathbf{k}}+\frac{1}{m_{k}} f^{2} Q_{k} \mathbf{e}_{\mathbf{k}},
\end{array}
$$

where $\epsilon=1 / \kappa$. Here $\epsilon$ represents the ratio of the vortex core thickness to the magnetic decay length. Many of the high-temperature superconducting materials have values of $\kappa$ around 50. We will be considering the limit $\kappa \rightarrow \infty, \epsilon \rightarrow 0$, in which the vortices appear as line singularities. 
2.1. Anisotropic scalings. It is convenient to introduce the following scalings (see [14], [2]) of the dependent and independent variables:

$$
\begin{gathered}
x_{i}=\left(m_{i}\right)^{-\frac{1}{2}} \tilde{x}_{i}, \\
H_{i}=\left(m_{i}\right)^{-\frac{1}{2}} \tilde{H}_{i}, \\
Q_{i}=\left(m_{i}\right)^{\frac{1}{2}} \tilde{Q}_{i},
\end{gathered}
$$

which have the useful property that the equations

$$
\begin{gathered}
\tilde{\mathbf{H}}=\tilde{\nabla} \wedge \tilde{\mathbf{Q}}, \\
\tilde{\nabla} \cdot \tilde{\mathbf{H}}=0
\end{gathered}
$$

and the phase of the order parameter, $\chi$, remain invariant $(\chi=\tilde{\chi})$. We introduce the following tensors for ease of notation:

$$
\begin{gathered}
\underline{\underline{D}}=\left(\begin{array}{ccc}
\left(m_{1}\right)^{-\frac{1}{2}} & 0 & 0 \\
0 & \left(m_{2}\right)^{-\frac{1}{2}} & 0 \\
0 & 0 & \left(m_{3}\right)^{-\frac{1}{2}}
\end{array}\right), \\
\underline{\underline{P}}=\left(\begin{array}{ccc}
\left(m_{1}\right)^{-1} & 0 & 0 \\
0 & \left(m_{2}\right)^{-1} & 0 \\
0 & 0 & \left(m_{3}\right)^{-1}
\end{array}\right), \\
\underline{\underline{N}}=\frac{1}{\alpha}\left(\begin{array}{ccc}
\sigma_{1} m_{1} & 0 & 0 \\
0 & \sigma_{2} m_{2} & 0 \\
0 & 0 & \sigma_{3} m_{3}
\end{array}\right) .
\end{gathered}
$$

The following identities also prove useful:

$$
\begin{aligned}
\epsilon_{k l m} D_{k r} D_{l s} D_{m t} & =\epsilon_{r s t}, \\
\epsilon_{k l m} P_{k r} P_{l s} P_{m t} & =\epsilon_{r s t},
\end{aligned}
$$

and similarly for the inverses $\underline{\underline{D}}^{-1}$ and $\underline{\underline{P}}^{-1}$.

With the scalings of (14) $\overline{-(16), \text { the }}$ anisotropic gauge-invariant time-dependent Ginzburg-Landau equations become

$$
\begin{array}{r}
-\frac{\partial f}{\partial t}+\tilde{\nabla}^{2} f=\frac{1}{\epsilon^{2}}\left(f^{3}-f\right)+f|\tilde{\mathbf{Q}}|^{2}, \\
f^{2} \Theta+\tilde{\nabla} \cdot\left(f^{2} \tilde{\mathbf{Q}}\right)=0, \\
-\tilde{\nabla} \wedge(\underline{\underline{P}}(\tilde{\nabla} \wedge \tilde{\mathbf{Q}}))=\underline{\underline{N}}\left(\frac{\partial \tilde{\mathbf{Q}}}{\partial t}+\tilde{\nabla} \Theta\right)+f^{2} \tilde{\mathbf{Q}} .
\end{array}
$$

Taking the divergence of (26) yields

$$
\tilde{\nabla} \cdot\left(f^{2} \tilde{\mathbf{Q}}\right)+\tilde{\nabla} \cdot\left(\underline{\underline{N}}\left(\frac{\partial \tilde{\mathbf{Q}}}{\partial t}+\tilde{\nabla} \Theta\right)\right)=0,
$$

which on combination with (25) gives

$$
f^{2} \Theta=\tilde{\nabla} \cdot\left(\underline{\underline{N}}\left(\frac{\partial \tilde{\mathbf{Q}}}{\partial t}+\tilde{\nabla} \Theta\right)\right) .
$$

Both of these results prove useful later. 
3. Self-induced vortex motion. Before we begin our analysis of vortex solutions of (24)-(26) we make two further assumptions concerning the remaining parameters. First we will consider only the case where $m_{1}=m_{2}$. This is a fairly realistic assumption for many layered crystal superconductors, such as $\mathrm{YBCuO}$ and $\mathrm{BiSCuO}$, where properties within a layer are isotropic. Second we take $1 / \alpha$ to be small. In the derivation of the isotropic time-dependent Ginzburg-Landau equations, from the Bordeen-Cooper-Schreiffer (BCS) theory, for the case of alloys with paramagnetic impurities, given by Gor'kov and Eliashburg [12] this parameter turns out to be $\frac{\epsilon^{2}}{12}$. Gor'kov and Kopnin [13] in their analysis of the motion of a vortex in these alloys take this parameter to be $o\left(\epsilon^{2}\right)$. In [6], the scaling $\frac{1}{\alpha}=O\left(\epsilon^{2}\right)$ was used, and this was found simply to modify the mobility coefficient of the vortices. In the anisotropic case the extra complication of having $\frac{1}{\alpha}=O\left(\epsilon^{2}\right)$ is great, and we consider only the simpler limit in which $\frac{1}{\alpha}=o\left(\epsilon^{2}\right)$. Hence we write

$$
\underline{\underline{N}}=\epsilon^{2} \delta \underline{\underline{n}}, \quad \text { where } \quad \delta=o(1) .
$$

Far field. We denote the outer solution away from the vortex cores by the subscript $o$. Equation (24) implies $f_{o}=1+O\left(\epsilon^{2}\right)$. Equations (25)-(27) give the leading order behavior of $\tilde{\mathbf{Q}}_{o}, \tilde{\mathbf{H}}_{o}$, and $\Theta_{o}$ as

$$
\begin{aligned}
& \tilde{\nabla} \wedge\left(\underline{\underline{P}}\left(\tilde{\nabla} \wedge \tilde{\mathbf{Q}}_{o}^{(0)}\right)\right)+\tilde{\mathbf{Q}}_{o}^{(0)}=\mathbf{0}, \\
& \Theta_{o}^{(0)}=-\tilde{\nabla} \cdot \tilde{\mathbf{Q}}_{o}^{(0)}=0, \\
& \tilde{\nabla} \wedge \tilde{\nabla} \wedge\left(\underline{\underline{P}} \tilde{\mathbf{H}}_{o}^{(0)}\right)+\tilde{\mathbf{H}}_{o}^{(0)}=\mathbf{0} .
\end{aligned}
$$

Equation (30) implies that

$$
\tilde{\mathbf{Q}}_{o}^{(0)}=-\tilde{\nabla} \wedge\left(\underline{\underline{P}} \tilde{\mathbf{H}}_{o}^{(0)}\right) .
$$

Inner region. We consider an inner region in the vicinity of one of the vortex cores. Let the vortex lie along the curve $\Gamma_{k}$, given by $\tilde{\mathbf{x}}=(\tilde{x}, \tilde{y}, \tilde{z})=\tilde{\mathbf{q}}(\tilde{s}, t)$. We define a local coordinate system $(\tilde{s}, \tilde{\rho}, \tilde{\theta}, T)$ by

$$
\begin{aligned}
\tilde{\mathbf{x}} & =\tilde{\mathbf{q}}(\tilde{s}, t)+\tilde{\rho} \cos \tilde{\theta} \tilde{\mathbf{n}}(\tilde{s}, t)+\tilde{\rho} \sin \tilde{\theta} \tilde{\mathbf{b}}(\tilde{s}, t), \\
t & =T,
\end{aligned}
$$

where $\tilde{s}$ is arclength, $\tilde{\mathbf{n}}(\tilde{s}, t)$ is the unit normal, and $\tilde{\mathbf{b}}(\tilde{s}, t)$ is the unit binormal to the curve $\Gamma_{k}$. We define the inner coordinates by introducing the stretched variable $\tilde{R}$ given by $\tilde{\rho}=\epsilon \tilde{R}$. The coordinate system so defined is not orthogonal away from the vortex line when the torsion of the vortex is nonzero. However, it is approximately orthogonal, and the following expressions for $\tilde{\nabla} \cdot \mathbf{B}, \tilde{\nabla}^{2} g, \tilde{\nabla} \wedge \mathbf{B}$, and $\frac{\partial}{\partial t}$ can be obtained:

$$
\begin{gathered}
\tilde{\nabla} g=\frac{\partial g}{\partial \tilde{s}} \mathbf{e}_{\tilde{\mathbf{s}}}+\frac{1}{\epsilon} \frac{\partial g}{\partial \tilde{R}} \mathbf{e}_{\tilde{\mathbf{R}}}+\frac{1}{\epsilon \tilde{R}} \frac{\partial g}{\partial \tilde{\theta}} \mathbf{e}_{\tilde{\theta}}+O(\epsilon), \\
\tilde{\nabla} \cdot \mathbf{B}=\frac{1}{\epsilon \tilde{R}}\left(\frac{\partial\left(\tilde{R} B_{\tilde{R}}\right)}{\partial \tilde{R}}+\frac{\partial B_{\tilde{\theta}}}{\partial \tilde{\theta}}\right)-\tilde{C}\left(B_{\tilde{R}} \cos \tilde{\theta}-B_{\tilde{\theta}} \sin \tilde{\theta}\right) \\
+\frac{\partial B_{\tilde{s}}}{\partial \tilde{s}}+\tilde{\tau} \frac{\partial B_{\tilde{s}}}{\partial \tilde{\theta}}+O(\epsilon),
\end{gathered}
$$




$$
\begin{gathered}
\tilde{\nabla}^{2} g=\frac{1}{\epsilon^{2} \tilde{R}}\left(\frac{\partial}{\partial \tilde{R}}\left(\tilde{R} \frac{\partial g}{\partial \tilde{R}}\right)+\frac{1}{\tilde{R}} \frac{\partial^{2} g}{\partial \tilde{\theta}^{2}}\right) \\
+\frac{\tilde{C}}{\epsilon}\left(\frac{1}{\tilde{R}} \frac{\partial g}{\partial \tilde{\theta}} \sin \tilde{\theta}-\frac{\partial g}{\partial \tilde{R}} \cos \tilde{\theta}\right)+O(1), \\
\tilde{\nabla} \wedge \mathbf{B}=\frac{1}{\epsilon}\left[\frac{1}{\tilde{R}}\left(\frac{\partial}{\partial \tilde{R}}\left(\tilde{R} B_{\tilde{\theta}}\right)-\frac{\partial B_{\tilde{R}}}{\partial \tilde{\theta}}\right) \mathbf{e}_{\tilde{\mathbf{s}}}+\frac{1}{\tilde{R}} \frac{\partial B_{\tilde{s}}}{\partial \tilde{\theta}} \mathbf{e}_{\tilde{\mathbf{R}}}-\frac{\partial B_{\tilde{s}}}{\partial \tilde{R}} \mathbf{e}_{\tilde{\theta}}\right] \\
+\left[\tilde{\tau}\left(\frac{\partial B_{\tilde{R}}}{\partial \tilde{\theta}}-\frac{\partial}{\partial \tilde{R}}\left(\tilde{R} B_{\tilde{\theta}}\right)\right)+\tilde{C} \cos \tilde{\theta} B_{\tilde{s}}+\frac{\partial B_{\tilde{R}}}{\partial \tilde{s}}\right] \mathbf{e}_{\tilde{\theta}} \\
\quad+\tilde{\tau} \tilde{R} \frac{\partial B_{\tilde{s}}}{\partial \tilde{R}} \mathbf{e}_{\tilde{\mathbf{s}}}+\left(\tilde{C} \sin \tilde{\theta} B_{\tilde{s}}-\frac{\partial B_{\tilde{\theta}}}{\partial \tilde{s}}\right) \mathbf{e}_{\tilde{\mathbf{R}}}+O(\epsilon), \\
\frac{\partial}{\partial t}=-\frac{1}{\epsilon}\left(\left(\tilde{\mathbf{v}} \cdot \mathbf{e}_{\tilde{\mathbf{R}}}\right) \frac{\partial}{\partial \tilde{R}}+\left(\tilde{\mathbf{v}} \cdot \mathbf{e}_{\tilde{\theta}}\right) \frac{1}{\tilde{R}} \frac{\partial}{\partial \tilde{\theta}}\right)+O(1),
\end{gathered}
$$

where $\tilde{C}$ is the curvature and $\tilde{\tau}$ is the torsion of the vortex line.

We denote the solution in the inner region by the subscript $i$. The presence of a vortex is defined by the following boundary conditions:

$$
f_{i} \rightarrow 0, \quad \tilde{\chi}_{i} \rightarrow N_{k} \tilde{\theta}, \quad \text { as } \tilde{R} \rightarrow 0 .
$$

We note that although the angle $\tilde{\theta}$ is not equivalent to the angle $\theta$, both increase by $2 \pi$ in a whole anticlockwise turn about the origin and hence leave $\psi$, the order parameter, single valued. The boundary condition for $\tilde{\chi}$ implies

$$
\tilde{\mathbf{Q}}_{i} \sim-\frac{N_{k}}{\epsilon \tilde{R}} \mathbf{e}_{\tilde{\theta}} \quad \text { as } \quad \tilde{R} \rightarrow 0,
$$

where $N_{k}$ is the vortex winding number. In light of (39)-(40) we make the following ansatz for the leading order behavior in the inner region:

$$
\begin{array}{r}
\tilde{\mathbf{Q}}_{i} \sim \frac{1}{\epsilon} \tilde{Q}_{i \tilde{\theta}}^{(0)}(\tilde{R}) \mathbf{e}_{\tilde{\theta}}+\cdots \\
f_{i} \sim f_{i}^{(0)}(\tilde{R})+\cdots
\end{array}
$$

When we substitute (41)-(42) into (17), (24), (26), and (27) and use the expressions obtained for the operators in the inner region, we find that

$$
\begin{array}{r}
\tilde{Q}_{i \theta}^{(0)}=-\frac{N_{k}}{\tilde{R}}, \\
f_{i}^{(0)^{\prime \prime}}+\frac{f_{i}^{(0)^{\prime}}}{\tilde{R}}-\frac{N_{k}^{2}}{\tilde{R}^{2}} f_{i}^{(0)}=f_{i}^{(0)^{3}}-f_{i}^{(0)}, \\
f_{i}^{(0)}(0)=0, \\
f_{i}^{(0)}(\infty)=1 .
\end{array}
$$

Thus the leading order solution for $f_{i}$ and $\tilde{\mathbf{Q}}_{i}$ (in the scaled coordinates) is exactly the same as that for the isotropic vortices [6]. The existence of a unique solution to (44)-(46) has been shown recently by Chen, Elliott, and Tang [7]. 
Far field. Matching (43) with the leading order far field solution gives the boundary condition

$$
\tilde{\mathbf{Q}}_{o}^{(0)} \sim-\frac{N_{k}}{\tilde{\rho}} \mathbf{e}_{\tilde{\theta}} \quad \text { as } \quad \tilde{\rho} \rightarrow 0 .
$$

This asymptotic behavior means that (32) may now be conveniently expressed in the form

$$
\tilde{\nabla} \wedge \tilde{\nabla} \wedge\left(\underline{\underline{P}} \tilde{\mathbf{H}}_{o}^{(0)}\right)+\tilde{\mathbf{H}}_{o}^{(0)}=\sum_{k} N_{k} \tilde{\boldsymbol{\delta}}
$$

where

$$
\tilde{\boldsymbol{\delta}}(\tilde{\mathbf{x}})=2 \pi \int_{\Gamma_{k}} \delta\left(\tilde{x}-\tilde{q}_{1}(\tilde{s})\right) \delta\left(\tilde{y}-\tilde{q}_{2}(\tilde{s})\right) \delta\left(\tilde{z}-\tilde{q}_{3}(\tilde{s})\right) \tilde{\mathbf{t}}(\tilde{s}) d \tilde{s} .
$$

In order to find the velocity of the vortex it is necessary to find an expression for $\tilde{\mathbf{Q}}_{o}^{(0)}$ as the vortex is approached and then to match this with first two terms of $\tilde{\mathbf{Q}}_{i}$. In the isotropic case examined in [6] we were able to solve (48) by means of a Green's function to obtain an integral expression for $\tilde{\mathbf{H}}_{o}^{(0)}$ and $\tilde{\mathbf{Q}}_{o}^{(0)}$ (equivalent to the Biot-Savart law in inviscid hydrodynamics). In the present case, however, such a solution is not available (in particular, the presence of the tensor $\underline{\underline{P}}$ means that there is coupling between the components of $\left.\mathbf{H}_{o}^{(0)}\right)$. However we can still go some way toward determining the behavior of $\tilde{\mathbf{Q}}_{o}^{(0)}$ as the vortex is approached.

In light of (17) we define a vector potential for $\tilde{\mathbf{Q}}_{o}^{(0)}$,

$$
\tilde{\mathbf{Q}}_{o}^{(0)}=-\tilde{\nabla} \wedge \mathbf{G} \quad \text { with } \quad \tilde{\nabla} \cdot \mathbf{G}=0 .
$$

Substitution of $\mathbf{G}$ into (32) gives the following equation and boundary condition for G,

$$
\begin{array}{r}
\tilde{\nabla} \wedge(\tilde{\nabla} \wedge \mathbf{G})+\tilde{\mathbf{H}}_{o}^{(0)}=\mathbf{0}, \\
\mathbf{G} \sim-N_{k} \log \tilde{\rho} \mathbf{e}_{\tilde{\mathbf{s}}} \quad \text { as } \quad \tilde{\rho} \rightarrow 0 .
\end{array}
$$

We aim to find the next term in the expansion of $\mathbf{G}$ as $\tilde{\rho} \rightarrow 0$. Since $\mathbf{H}_{o}^{(0)}=O(\log \tilde{\rho})$ as $\tilde{\rho} \rightarrow 0$, this term will not contribute to the asymptotic behavior of $\mathbf{G}$ until $O\left(\tilde{\rho}^{2} \log \tilde{\rho}\right)$. Hence we can obtain the asymptotic behavior of $\mathbf{G}$ up to this order by solving the system

$$
\begin{array}{r}
\tilde{\nabla} \wedge(\tilde{\nabla} \wedge \mathbf{F})=-\tilde{\nabla}^{2} \mathbf{F}=\mathbf{0}, \\
\mathbf{F} \sim-N_{k} \log \tilde{\rho} \mathbf{e}_{\tilde{\mathbf{s}}} \quad \text { as } \quad \tilde{\rho} \rightarrow 0 .
\end{array}
$$

The solution to (53) and (54) given in Batchelor [1] is

$$
\mathbf{F}=\frac{1}{2} \sum_{k} N_{k} \int_{\Gamma_{k}} \frac{1}{Z} d \tilde{\mathbf{x}}^{\prime}
$$

where $Z=\left|\tilde{\mathbf{x}}-\tilde{\mathbf{x}}^{\prime}\right|$. Expanding $\mathbf{F}$ as $\tilde{\rho} \rightarrow 0$ we find [16]

$$
\mathbf{F} \sim-N_{k} \log \tilde{\rho} \mathbf{e}_{\tilde{\mathbf{s}}}+\frac{N_{k} \tilde{C}}{2} \tilde{\rho} \log \tilde{\rho} \cos \tilde{\theta} \mathbf{e}_{\tilde{\mathbf{s}}}+O(\tilde{\rho}) .
$$

Now, since $\mathbf{F}-\mathbf{G}=o(\tilde{\rho} \log \tilde{\rho})$ as $\tilde{\rho} \rightarrow 0$ we find

$$
\mathbf{Q}_{o}^{(0)} \sim-\frac{N_{k}}{\tilde{\rho}} \mathbf{e}_{\tilde{\theta}}+\frac{\tilde{C} N_{k}}{2} \log \tilde{\rho} \tilde{\mathbf{b}}+\cdots \quad \text { as } \quad \tilde{\rho} \rightarrow 0 .
$$


Inner region. It is now apparent how to proceed with the inner expansion,

$$
\begin{array}{r}
f_{i}=f_{i}^{(0)}(\tilde{R})+\epsilon \log \epsilon f_{i}^{(1)}+\cdots, \\
\tilde{\mathbf{Q}}_{i}=-\frac{N_{k}}{\epsilon \tilde{R}} \mathbf{e}_{\tilde{\theta}}+\log \epsilon \tilde{\mathbf{Q}}_{i}^{(1)}+\cdots, \\
\tilde{\mathbf{v}}_{i}=\log \epsilon \tilde{\mathbf{v}}_{i}^{(0)}+\tilde{\mathbf{v}}_{i}^{(1)}+\cdots, \\
\Theta_{i}=\frac{\delta \log \epsilon}{\epsilon} \Theta_{i}^{(0)}+\cdots, \\
\tilde{\mathbf{H}}_{i}=\log \epsilon \tilde{\mathbf{H}}_{i}^{(0)}+\cdots .
\end{array}
$$

The scaling of $\Theta_{i}$ here is worth further discussion. The scaling given is the only consistent balance of terms in (28), corresponding to a balance between the first and second terms. However, $\Theta_{i}$ must satisfy the the boundary condition

$$
\Theta_{i} \rightarrow-\frac{1}{\epsilon}\left(\left(\tilde{\mathbf{v}} \cdot \mathbf{e}_{\tilde{\mathbf{R}}}\right) \frac{\partial}{\partial \tilde{R}}+\left(\tilde{\mathbf{v}} \cdot \mathbf{e}_{\tilde{\theta}}\right) \frac{1}{\tilde{R}} \frac{\partial}{\partial \tilde{\theta}}\right) \tilde{\chi} \quad \text { as } \quad \tilde{R} \rightarrow 0,
$$

seemingly a contradiction since the right-hand side here is $O\left(\frac{\log \epsilon}{\epsilon}\right)$ and corresponds to a balance between the second and third terms in (28). In fact, to satisfy (63) we need to introduce an inner-inner region defined by

$$
\tilde{R}=\delta^{1 / 4} \tilde{r}
$$

in which

$$
\begin{array}{r}
f_{i i}=D \delta^{1 / 4} \tilde{r}+\cdots, \\
\tilde{\mathbf{Q}}_{i i}=-\frac{1}{\delta^{1 / 4} \epsilon \tilde{r}} \mathbf{e}_{\tilde{\theta}}+\cdots, \\
\Theta_{i i}=\frac{\log \epsilon}{\delta^{1 / 4} \epsilon} \Theta_{i i}^{(0)}+\cdots,
\end{array}
$$

In this region all three terms in (28) balance, and the boundary condition (63) can be satisfied. As $\tilde{r} \rightarrow \infty$ in this inner-inner region we find

$$
\Theta_{i i}^{(0)} \sim O\left(\frac{1}{\tilde{r}^{5}}\right)
$$

matching into the scaling given in (61).

It is now possible to exactly parallel the analysis in [6], and we refer the reader to that reference for the details.

It is necessary for $\tilde{\mathbf{v}}^{(0)}$ to be in the normal direction if $\tilde{\mathbf{Q}}_{i}^{(1)}$ is to match to $\tilde{\mathbf{Q}}_{o}^{(0)}$; hence we write

$$
\tilde{\mathbf{v}}^{(0)}=U_{0} \tilde{\mathbf{n}} .
$$

Proceeding to the next order in (24)-(28) we obtain a system of inhomogeneous linear partial differential equations. There are nontrivial solutions to the homogeneous versions of these equations, and therefore, by the Fredholm alternative, there is a solution to the first order equations if and only if a certain solvability condition is satisfied. After some calculation this condition is found to be

$$
\lim _{\tilde{R} \rightarrow \infty} \tilde{\mathbf{Q}}_{i}^{(1)}=-\frac{U_{0} \beta}{2 N_{k}} \tilde{\mathbf{b}},
$$


where

$$
\beta=\int_{0}^{\infty} \tilde{R} f_{i}^{(0)^{\prime 2}} d \tilde{R}
$$

(It should be noted that this form of $\beta$ differs from that in [6] because we have been able to neglect the contribution to $\beta$ from the scalar potential $\Theta_{i}$ since $\delta=o(1)$.)

Matching. We complete this part of the analysis by matching $\tilde{\mathbf{Q}}_{i}^{(1)}$ with $\tilde{\mathbf{Q}}_{o}^{(0)}$ using (68) and (57), and following the matching principle of Van Dyke [17]. By inspection we see

$$
\tilde{\mathbf{v}}^{(0)}=-\frac{\tilde{C} N_{k}^{2}}{\beta} \tilde{\mathbf{n}}
$$

Hence, the self-induced velocity of a curved vortex line in the anisotropic GinzburgLandau model is proportional to curvature and in the normal direction, exactly as in the isotropic case, but in the scaled coordinates (14)-(16). Let us now return to the physical variables to see the effect that the anisotropy has on the motion.

Unscaled coordinates. We start by noting that the choice of the $x$-axis is arbitrary provided it remains in the plane of isotropy. Hence, we can choose a set of axes such that the tangent to the vortex curve lies in the $x-z$ plane at the point on its length $\mathbf{x}=\mathbf{q}\left(s_{0}, t\right)$, where $\mathbf{q}(s, t)$ gives the position of the vortex line. We may expand $\mathbf{q}(s, t)$ about the point given by $s=s_{0}$,

$$
\mathbf{q}(s, t)=\mathbf{q}\left(s_{0}, t\right)+\left(s-s_{0}\right) \mathbf{t}\left(s_{0}, t\right)+\frac{\left(s-s_{0}\right)^{2}}{2} C \mathbf{n}\left(s_{0}, t\right)+\cdots \quad .
$$

Since $\mathbf{t}\left(s_{0}, t\right)$, the unit tangent vector, lies in the $x-z$ plane, it can be expressed as

$$
\mathbf{t}\left(s_{0}, t\right)=(\sin \alpha, 0, \cos \alpha) .
$$

The unit normal at this point $\mathbf{n}\left(s_{0}, t\right)$ is orthogonal to the tangent and so without loss of generality can be written as

$$
\mathbf{n}\left(s_{0}, t\right)=(\sin \phi \cos \alpha, \cos \phi,-\sin \phi \sin \alpha) .
$$

The unit binormal vector is determined by the relation $\mathbf{b}=\mathbf{t} \wedge \mathbf{n}$ and hence

$$
\mathbf{b}\left(s_{0}, t\right)=(-\cos \alpha \cos \phi, \sin \phi, \sin \alpha \cos \phi) .
$$

After writing $\mathbf{q}\left(s_{0}, t\right)=(a, b, c)$ we can express each component of $\mathbf{q}(s, t)$ as a Taylor series about the point given by $s_{0}$. It is then easy to write down the representation of the vortex in terms of the scaled coordinates,

$$
\begin{array}{r}
\tilde{q}_{1}=m_{1}^{\frac{1}{2}} a+m_{1}^{\frac{1}{2}}\left(s-s_{0}\right) \sin \alpha+m_{1}^{\frac{1}{2}} \frac{\left(s-s_{0}\right)^{2}}{2} C \sin \phi \cos \alpha+\cdots, \\
\tilde{q}_{2}=m_{1}^{\frac{1}{2}} b+m_{1}^{\frac{1}{2}} \frac{\left(s-s_{0}\right)^{2}}{2} C \cos \phi+\cdots, \\
\tilde{q}_{3}=m_{3}^{\frac{1}{2}} c+m_{3}^{\frac{1}{2}}\left(s-s_{0}\right) \cos \alpha-m_{3}^{\frac{1}{2}} \frac{\left(s-s_{0}\right)^{2}}{2} C \sin \phi \sin \alpha+\cdots
\end{array}
$$

From (75)-(77) we calculate $\tilde{\mathbf{t}}\left(s_{0}, t\right)$ by noting

$$
\tilde{\mathbf{t}}=\frac{d \tilde{\mathbf{q}}}{d \tilde{s}} \quad \text { and } \quad \frac{d \tilde{s}}{d s}=\left|\frac{d \tilde{\mathbf{q}}}{d s}\right| .
$$


It then follows that

$$
\tilde{\mathbf{t}}\left(s_{0}, t\right)=\frac{1}{\left(m_{1} \sin ^{2} \alpha+m_{3} \cos ^{2} \alpha\right)^{\frac{1}{2}}}\left(m_{1}^{\frac{1}{2}} \sin \alpha, 0, m_{3}^{\frac{1}{2}} \cos \alpha\right) .
$$

In a similar way, $\tilde{C} \tilde{\mathbf{n}}$ is calculated from

$$
\tilde{C} \tilde{\mathbf{n}}=\frac{d^{2} \tilde{\mathbf{q}}}{d \tilde{s}^{2}}
$$

to give

$$
\begin{aligned}
& (\tilde{C} \tilde{\mathbf{n}})_{1}\left(s_{0}, t\right)=\frac{C m_{1}^{\frac{1}{2}} m_{3} \sin \phi \cos \alpha}{\left(m_{1} \sin ^{2} \alpha+m_{3} \cos ^{2} \alpha\right)^{2}}, \\
& (\tilde{C} \tilde{\mathbf{n}})_{2}\left(s_{0}, t\right)=\frac{C m_{1}^{\frac{1}{2}} \cos \phi}{\left(m_{1} \sin ^{2} \alpha+m_{3} \cos ^{2} \alpha\right)}, \\
& (\tilde{C} \tilde{\mathbf{n}})_{3}\left(s_{0}, t\right)=\frac{-C m_{1} m_{3}^{\frac{1}{2}} \sin \phi \sin \alpha}{\left(m_{1} \sin ^{2} \alpha+m_{3} \cos ^{2} \alpha\right)^{2}} .
\end{aligned}
$$

Referring back to (70) it is now possible to write down $\tilde{\mathbf{v}}^{(0)}$ in terms of the vortex curvature and orientation in the unscaled frame using the value of $\tilde{C} \tilde{\mathbf{n}}$ in (81)-(83). It is clear that velocity should scale in the same way as position and hence we use (14) to write down the velocity in the unscaled frame,

$$
\begin{gathered}
v_{1}^{(0)}=\frac{1}{m_{1}^{\frac{1}{2}}} \tilde{v}_{1}^{(0)}=-\frac{N_{k}^{2} m_{3} C \sin \phi \cos \alpha}{\beta\left(m_{1} \sin ^{2} \alpha+m_{3} \cos ^{2} \alpha\right)^{2}} \\
v_{2}^{(0)}=\frac{1}{m_{1}^{\frac{1}{2}}} \tilde{v}_{1}^{(0)}=-\frac{N_{k}^{2} C \cos \phi}{\beta\left(m_{1} \sin ^{2} \alpha+m_{3} \cos ^{2} \alpha\right)} \\
v_{3}^{(0)}=\frac{1}{m_{3}^{\frac{1}{2}}} \tilde{v}_{1}^{(0)}=\frac{N_{k}^{2} m_{1} C \sin \phi \sin \alpha}{\beta\left(m_{1} \sin ^{2} \alpha+m_{3} \cos ^{2} \alpha\right)^{2}}
\end{gathered}
$$

We note that a line is unaffected by a velocity tangential to itself, and so we require only the components of velocity in the normal and binormal directions to fully specify its movement. The effective velocity of the vortex line, given on calculation of these two components, is

$$
\mathbf{v}^{(0)}=-\frac{N_{k}^{2} C \mathbf{n}}{\beta\left(m_{1} \sin ^{2} \alpha+m_{3} \cos ^{2} \alpha\right)} .
$$

Thus we see that the vortex still moves only in the normal direction and is still proportional to the curvature, but the constant of proportionality is a function of the angle the tangent to the vortex line makes with the $c$-axis.

4. Homogenization of a system of closely packed vortices. So far we have been considering a system of vortices which are separated by distances of order one. Let us now consider a situation in which the vortices are separated by distances of order $\zeta$, where $\epsilon^{\frac{1}{2}} \ll \zeta \ll 1 /|\log \epsilon|^{\frac{1}{2}}$, as $\epsilon \rightarrow 0$. The magnetic field will then be $O\left(1 / \zeta^{2}\right)$ due to the increased density of vortices. 
We consider the case in which $N_{i}=1$, for all $i$, since vortices with higher vortex numbers are thought to be unstable. We define an averaged vorticity, as in [4], by

$$
\tilde{\boldsymbol{\omega}}_{\eta}(\tilde{\mathbf{x}})=\int_{\left|\tilde{x}-\tilde{x}^{\prime}\right|<\frac{\eta}{2}} \int_{\left|\tilde{y}-\tilde{y}^{\prime}\right|<\frac{\eta}{2}} \int_{\left|\tilde{z}-\tilde{z}^{\prime}\right|<\frac{\eta}{2}} \frac{1}{\eta^{3}} \sum_{i} \tilde{\delta}_{i}\left(\tilde{\mathbf{x}}_{i}^{\prime}\right) d \tilde{x}^{\prime} d \tilde{y}^{\prime} d \tilde{z}^{\prime}
$$

and consider the limit as $\eta \rightarrow 0$ with $\eta \gg \zeta$ so that there are many vortices enclosed within the region of integration. Since $\tilde{\boldsymbol{\omega}}_{\eta}$ will also be $O\left(1 / \zeta^{2}\right)$, we define

$$
\tilde{\boldsymbol{\omega}}=\lim _{\eta \rightarrow 0} \zeta^{2} \tilde{\boldsymbol{\omega}}_{\eta}
$$

Assuming $\tilde{\boldsymbol{\omega}}$ to vary on an order one scale, it is not difficult to show formally that with $\tilde{\mathbf{H}}_{o}$ and $\tilde{\mathbf{Q}}_{o}$ expanded as

$$
\begin{aligned}
\tilde{\mathbf{H}}_{o} & =\frac{\tilde{\mathbf{H}}_{o}^{(0)}}{\zeta^{2}}+\cdots, \\
\tilde{\mathbf{Q}}_{o} & =\frac{\tilde{\mathbf{Q}}_{o}^{(0)}}{\zeta^{2}}+\cdots,
\end{aligned}
$$

the leading order behavior is governed by

$$
\begin{gathered}
\tilde{\nabla} \wedge\left(\tilde{\nabla} \wedge\left(\underline{\underline{P}} \tilde{\mathbf{H}}_{o}^{(0)}\right)\right)+\tilde{\mathbf{H}}_{o}^{(0)}=\tilde{\boldsymbol{\omega}} \\
\tilde{\nabla} \cdot \tilde{\mathbf{H}}_{o}^{(0)}=0 \\
\tilde{\mathbf{Q}}_{o}^{(0)}=-\tilde{\nabla} \wedge\left(\underline{\underline{P}} \tilde{\mathbf{H}}_{o}^{(0)}\right) .
\end{gathered}
$$

Once the individual vortices have been averaged to form a vorticity, it is necessary to add an equation describing the conservation of vorticity. Such an equation can be derived exactly as in the isotropic case, and is given by

$$
\frac{\partial \tilde{\boldsymbol{\omega}}}{\partial t}+\tilde{\nabla} \wedge(\tilde{\boldsymbol{\omega}} \wedge \tilde{\mathbf{v}})=\mathbf{0},
$$

where $\tilde{\mathbf{v}}$ is the velocity of the vortex lines. To close the model it is necessary to derive an expression for this velocity, which will again require the consideration of an inner region in the vicinity of each vortex. In fact, to be systematic, we should also consider the intermediate region in which lengths are scaled with $\zeta$ and the vortices again appear as discrete lines.

Intermediate region. We rescale lengths with $\zeta$ in the vicinity of an arbitrary point $\tilde{\mathbf{x}}_{0}$ so that $\tilde{\mathbf{x}}-\tilde{\mathbf{x}}_{0}=\zeta \tilde{\mathbf{X}}$. Thus, on this scale the vortices are $O(1)$ distances apart. In order to match with the outer expansion, the fields $\tilde{\mathbf{H}}$ and $\tilde{\mathbf{Q}}$ are expanded as

$$
\begin{aligned}
\tilde{\mathbf{H}}_{i n t} & =\frac{\tilde{\mathbf{H}}_{i n t}^{(0)}}{\zeta^{2}}+\cdots, \\
\tilde{\mathbf{Q}}_{i n t} & =\frac{\tilde{\mathbf{Q}}_{i n t}^{(0)}}{\zeta^{2}}+\cdots .
\end{aligned}
$$


Equations (17), (18), (24), (26), and (27), when written in terms of the intermediate variables, give the leading order behavior

$$
\begin{gathered}
f_{i n t}^{(0)}=1, \\
\tilde{\nabla} \wedge\left(\underline{\underline{P}} \tilde{\mathbf{H}}_{i n t}^{(0)}\right)=\mathbf{0}, \\
\tilde{\nabla} \cdot \tilde{\mathbf{H}}_{i n t}^{(0)}=0, \\
\tilde{\nabla} \cdot \tilde{\mathbf{Q}}_{i n t}^{(0)}=0, \\
\tilde{\nabla} \wedge \tilde{\mathbf{Q}}_{i n t}^{(0)}=\mathbf{0} .
\end{gathered}
$$

When the leading order inner behavior near each vortex, $\tilde{\mathbf{Q}}_{i}^{(0)}=-\mathbf{e}_{\tilde{\theta}} / \epsilon \tilde{R}$, is matched into this region, we find that it is $O\left(\frac{1}{\zeta}\right)$. Thus, the individual vortices can be distinguished in this region only at first order, and the leading order $\tilde{\mathbf{H}}_{i n t}$ and $\tilde{\mathbf{Q}}_{i n t}$ are simply constant, being the average "background" value generated by the ensemble of vortices. Matching with the outer region,

$$
\begin{aligned}
\tilde{\mathbf{Q}}_{i n t}^{(0)} & =\tilde{\mathbf{Q}}_{o}^{(0)}\left(\tilde{\mathbf{x}}_{0}\right), \\
\tilde{\mathbf{H}}_{i n t}^{(0)} & =\tilde{\mathbf{H}}_{o}^{(0)}\left(\tilde{\mathbf{x}}_{0}\right) .
\end{aligned}
$$

Inner region. In the inner region the expansions given previously are modified since they must match with the large "background" $\tilde{\mathbf{H}}$ and $\tilde{\mathbf{Q}}$ fields in the outer region:

$$
\begin{array}{r}
f_{i}=f_{i}^{(0)}(\tilde{R})+\frac{\epsilon}{\zeta^{2}} f_{i}^{(1)}+\cdots, \\
\tilde{\mathbf{Q}}_{i}=-\frac{1}{\epsilon \tilde{R}} \mathbf{e}_{\tilde{\theta}}+\frac{1}{\zeta^{2}} \tilde{\mathbf{Q}}_{i}^{(1)}+\cdots, \\
\Theta=o\left(\frac{1}{\zeta^{2}}\right), \\
\tilde{\mathbf{H}}_{i}=\frac{\tilde{\mathbf{H}}_{i}^{(0)}}{\zeta^{2}}+\cdots, \\
\tilde{\mathbf{v}}=\frac{\tilde{\mathbf{v}}^{(0)}}{\zeta^{2}}+\cdots
\end{array}
$$

The calculation now proceeds exactly as in the isotropic case [6]. Again there is a solvability condition for the first order equations, which after some manipulation yields the following law for the velocity of the vortex:

$$
\tilde{\mathbf{v}}^{(0)}=-\frac{2}{\beta} \tilde{\mathbf{Q}}_{o}^{(0)} \wedge \tilde{\mathbf{t}}
$$

with $\beta$ given by (69).

Thus we arrive at the following anisotropic mean-field model, in scaled coordinates:

$$
\tilde{\nabla} \wedge\left(\tilde{\nabla} \wedge\left(\underline{\underline{P}} \tilde{\mathbf{H}}_{o}^{(0)}\right)\right)+\tilde{\mathbf{H}}_{o}^{(0)}=\tilde{\boldsymbol{\omega}}
$$




$$
\begin{gathered}
\tilde{\nabla} \cdot \tilde{\mathbf{H}}_{o}^{(0)}=0, \\
\frac{\partial \tilde{\boldsymbol{\omega}}}{\partial t}+\tilde{\nabla} \wedge\left(\tilde{\boldsymbol{\omega}} \wedge \tilde{\mathbf{v}}^{(0)}\right)=\mathbf{0}, \\
\tilde{\mathbf{v}}^{(0)}=\frac{2}{\beta}\left(\tilde{\nabla} \wedge\left(\underline{\underline{P}}_{\tilde{\mathbf{H}}_{o}^{(0)}}\right)\right) \wedge \frac{\tilde{\boldsymbol{\omega}}}{|\tilde{\boldsymbol{\omega}}|} .
\end{gathered}
$$

Unscaled coordinates. From (106) we derive the leading order velocity of the vortex line in the unstretched coordinates, $\mathbf{v}^{(0)}$, in terms of $\mathbf{Q}_{o}^{(0)}$ and the angle $\mathbf{t}$ makes with the $c$-axis, $\alpha$ :

$$
\mathbf{v}^{(0)}=\frac{2}{\beta\left(m_{1} \sin ^{2} \alpha+m_{3} \cos ^{2} \alpha\right)^{\frac{1}{2}}}\left(\begin{array}{c}
-m_{3} Q_{2} \cos \alpha \\
m_{3} Q_{1} \cos \alpha-m_{1} Q_{3} \sin \alpha \\
m_{1} Q_{2} \sin \alpha Q
\end{array}\right)^{T}
$$

We can rewrite this as

$$
\mathbf{v}^{(0)}=-\frac{2}{\beta\left(m_{1} \sin ^{2} \alpha+m_{3} \cos ^{2} \alpha\right)^{\frac{1}{2}}} \mathbf{Q} \wedge\left(\underline{\underline{P}}^{-1} \mathbf{t}\right) .
$$

An anisotropic London equation can be derived from (48) by rescaling. It is fairly easy to show that

$$
\left(\tilde{\nabla} \wedge\left(\tilde{\nabla} \wedge\left(\underline{\underline{P}}_{o}\right)\right)+\tilde{\mathbf{H}}_{o}\right)_{j}=\sqrt{m_{j}}\left(\nabla \wedge\left(\underline{\underline{P}}^{-1}\left(\nabla \wedge \mathbf{H}_{o}\right)\right)+\mathbf{H}_{o}\right)_{j}
$$

by appealing to (22) and (23). The scaled tangent vector maybe written as

$$
\tilde{\mathbf{t}}(\tilde{s})=\frac{d \tilde{\mathbf{q}}}{d s} \frac{d s}{d \tilde{s}},
$$

giving

$$
\tilde{\mathbf{t}}(\tilde{s}) d \tilde{s}=\left(\sqrt{m_{1}} t_{1}, \sqrt{m_{1}} t_{2}, \sqrt{m_{3}} t_{3}\right) d s .
$$

On substitution of (113) and (115) into (48), noting that $\delta\left(\sqrt{m_{1}} x\right)=\left(1 / \sqrt{m_{1}}\right) \delta(x)$, it is found that the unscaled London equation takes the form

$$
\nabla \wedge\left(\underline{\underline{P}}^{-1}\left(\nabla \wedge \mathbf{H}_{o}^{(0)}\right)\right)+\mathbf{H}_{o}^{(0)}=\frac{1}{m_{1} \sqrt{m_{3}}} \sum_{i} \boldsymbol{\delta}_{\boldsymbol{i}}=\sum_{i} \boldsymbol{\delta}_{\boldsymbol{i}},
$$

where

$$
\boldsymbol{\delta}_{\boldsymbol{i}}(\mathbf{x})=2 \pi \int_{\Gamma_{i}} \delta\left(x-q_{1}(s)\right) \delta\left(y-q_{2}(s)\right) \delta\left(z-q_{3}(s)\right) \mathbf{t}(s) d s .
$$

The unscaled vorticity is therefore defined by

$$
\begin{gathered}
\boldsymbol{\omega}_{\eta}=\int_{\left|x-x^{\prime}\right|<\frac{\eta}{2 m_{1}^{\frac{1}{2}}}} \int_{\left|y-y^{\prime}\right|<\frac{\eta}{2 m_{1}^{\frac{1}{2}}}} \int_{\left|z-z^{\prime}\right|<\frac{\eta}{2 m_{3}^{\frac{1}{2}}}} \frac{1}{\eta^{3}} \sum_{i} \delta_{i} d x^{\prime} d y^{\prime} d z^{\prime}, \\
\boldsymbol{\omega}=\lim _{\eta \rightarrow 0} \zeta^{2} \boldsymbol{\omega}_{\eta} \quad \text { with } \quad \zeta \ll \eta .
\end{gathered}
$$


The homogeneous model is arrived at in the same manner as for the scaled case, and is

$$
\begin{array}{r}
\nabla \wedge\left(\underline{\underline{P}}^{-1}\left(\nabla \wedge \mathbf{H}_{o}^{(0)}\right)\right)+\mathbf{H}_{o}^{(0)}=\boldsymbol{\omega}, \\
\nabla \cdot \mathbf{H}_{o}^{(0)}=0, \\
\frac{\partial \boldsymbol{\omega}}{\partial t}+\nabla \wedge(\boldsymbol{\omega} \wedge \mathbf{v})=\mathbf{0}, \\
\mathbf{Q}_{o}^{(0)}=-\underline{\underline{P}}^{-1}\left(\nabla \wedge \mathbf{H}_{o}^{(0)}\right),
\end{array}
$$

with $\mathbf{v}$ given by (112). It is a straightforward matter to show the definitions of $\boldsymbol{\omega}$ and $\tilde{\boldsymbol{\omega}}$ imply that

$$
\omega_{i}=\left(m_{i}\right)^{-\frac{1}{2}} \tilde{\omega}_{i} .
$$

It then follows that the two models (120)-(123) and (107)-(92) and (109) are equivalent.

Externally driven vortex motion. For completeness we now consider a system of individual vortices lying along curves $\Gamma_{i}$, where the dominant component of velocity of any vortex is not self-induced but results from other vortices. Whether such a situation arises depends on the relative sizes of the current $\nabla \wedge \mathbf{H}$ and the curvature $C$. In particular, when $C_{i} \log \kappa \ll|\nabla \wedge \mathbf{H}|$ the motion of the $i$ th vortex is externally driven. Where this is the case a derivation of the velocity law for the $i$ th vortex can be made in a manner almost exactly identical to the previous section. Indeed it is found that

$$
\mathbf{v}_{i}^{(0)}(\mathbf{q}(s, t))=-\left.\frac{2}{\beta\left(m_{1} \sin ^{2} \alpha+m_{3} \cos ^{2} \alpha\right)^{\frac{1}{2}}} \mathbf{Q}_{\mathbf{i}} \wedge\left(\underline{\underline{P}}^{-1} \mathbf{t}_{i}\right)\right|_{\mathbf{x}=\mathbf{q}(\mathbf{s}, \mathbf{t})},
$$

where

$$
\mathbf{Q}_{i}=\underline{\underline{P}}^{-1}\left(\nabla \wedge \mathbf{H}_{i}\right)
$$

and

$$
\begin{aligned}
\nabla \wedge\left(\underline{\underline{P}}^{-1}\left(\nabla \wedge \mathbf{H}_{i}\right)\right)+\mathbf{H}_{i} & =\sum_{j \neq i} \delta_{j}, \\
\nabla \cdot \mathbf{H}_{i} & =0 .
\end{aligned}
$$

\section{Examples.}

5.1. A planar vortex curve moving under curvature. In this section we consider the motion of a vortex moving under curvature and lying along a closed planar curve. Consider the normal to the plane of the curve to make an angle $\theta$ with the $z$-axis. Performing a rotation of coordinates such that the $\bar{z}$-axis is now perpendicular to the plane of the vortex (see Figure 1) we may in general parameterize the position of the vortex line by

$$
\mathbf{q}(u, t)=\bar{X}(u, t) \mathbf{e}_{\overline{\mathbf{x}}}+\bar{Y}(u, t) \mathbf{e}_{\overline{\mathbf{y}}} .
$$

Thus the angle $\alpha$ that the vortex makes with the $z$-axis is given by

$$
\cos ^{2} \alpha=\frac{\bar{X}_{u}^{2} \sin ^{2} \theta}{\bar{X}_{u}^{2}+\bar{Y}_{u}^{2}}, \quad \sin ^{2} \alpha=\frac{\bar{X}_{u}^{2} \cos ^{2} \theta+\bar{Y}_{u}^{2}}{\bar{X}_{u}^{2}+\bar{Y}_{u}^{2}} .
$$




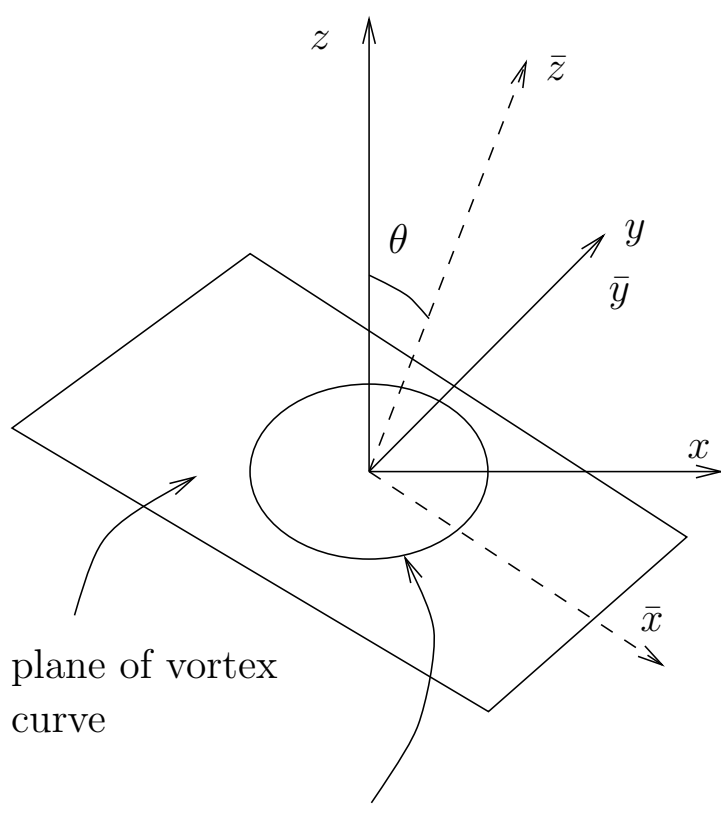

vortex curve

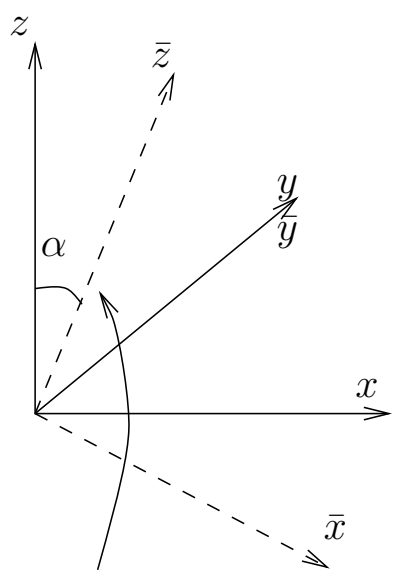

direction of vortex line

(a)

(b)

FIG. 1. The configurations under consideration: (a) a planar curve moving under curvature and (b) a vortex at an angle to the axes of anisotropy.

Inserting the parameterization of the vortex line (129) into the velocity law for motion under curvature (87) and scaling time suitably, we obtain the following coupled degenerate partial differential equations:

$$
\begin{gathered}
\bar{X}_{t}-\frac{\left(\bar{X}_{u}^{2}+\bar{Y}_{u}^{2}\right)^{1 / 2}}{b \bar{X}_{u}^{2}+m_{1} \bar{Y}_{u}^{2}}\left(\frac{\bar{X}_{u}}{\left(\bar{X}_{u}^{2}+\bar{Y}_{u}^{2}\right)^{1 / 2}}\right)_{u}=0 \\
\bar{Y}_{t}-\frac{\left(\bar{X}_{u}^{2}+\bar{Y}_{u}^{2}\right)^{1 / 2}}{b \bar{X}_{u}^{2}+m_{1} \bar{Y}_{u}^{2}}\left(\frac{\bar{Y}_{u}}{\left(\bar{X}_{u}^{2}+\bar{Y}_{u}^{2}\right)^{1 / 2}}\right)_{u}=0
\end{gathered}
$$

where

$$
b=\left(m_{1}+\frac{1}{m_{1}^{2}} \sin ^{2} \theta\right) .
$$

Exact solutions. In the scaled frame the equation of motion of a vortex moving under curvature is, with the same scaling of time as above,

$$
\tilde{\mathbf{v}}=-\tilde{C} \tilde{\mathbf{n}} .
$$

Thus an initially circular ring in the scaled frame, radius $\tilde{r}_{0}$, will contract according to the law $\tilde{r}^{2}=\tilde{r}_{0}^{2}-2 t$, where $\tilde{r}$ is the radius of the vortex. When the vortex lies in a plane at an angle $\tilde{\theta}$ to the $z$-axis this may be rewritten in the form

$$
(\tilde{x} \cos \tilde{\theta}-\tilde{z} \sin \tilde{\theta})^{2}+\tilde{y}=\tilde{r}_{0}^{2}-2 t,
$$


which gives the following exact formula for the contraction of an ellipse in the unscaled frame:

$$
\tilde{b}(x \cos \theta-z \sin \theta)^{2}+m_{1} y^{2}=\tilde{r}_{0}^{2}-2 t,
$$

where

$$
\tilde{\theta}=\arctan \left(m_{1}^{3 / 2} \tan \theta\right) \quad \text { and } \quad \tilde{b}=\left(m_{1} \cos ^{2} \tilde{\theta}+\frac{1}{m_{1}^{2}} \sin ^{2} \tilde{\theta}\right) .
$$

It is worth remarking here that $\theta$ is the angle made by the normal to the plane of the ellipse to the $z$-axis.

Numerical solution. Following Decklenick and Dziuk [9], who consider motion under curvature in an isotropic medium, we multiply by a test function $\phi(u)$ and integrate over the length of the curve to arrive at the weak formulation

$$
\begin{gathered}
\int \phi\left(\frac{b \bar{X}_{u}^{2}+m_{1} \bar{Y}_{u}^{2}}{\left(\bar{X}_{u}^{2}+m_{1} \bar{Y}_{u}^{2}\right)^{1 / 2}}\right) \bar{X}_{t} d u+\int \phi_{u}\left(\frac{\bar{X}_{u}}{\left(\bar{X}_{u}^{2}+\bar{Y}_{u}^{2}\right)^{1 / 2}}\right) d u=0, \\
\int \phi\left(\frac{b \bar{X}_{u}^{2}+m_{1} \bar{Y}_{u}^{2}}{\left(\bar{X}_{u}^{2}+m_{1} \bar{Y}_{u}^{2}\right)^{1 / 2}}\right) \bar{Y}_{t} d u+\int \phi_{u}\left(\frac{\bar{Y}_{u}}{\left(\bar{X}_{u}^{2}+\bar{Y}_{u}^{2}\right)^{1 / 2}}\right) d u=0 .
\end{gathered}
$$

We now restrict our attention to piecewise linear continuous functions on a grid $s_{j}=$ $j h(j=1,2, \ldots, n)$ such that

$$
\bar{X}_{h}=\sum \bar{X}_{j}(t) \phi_{j}(u) \quad \text { and } \quad \bar{Y}_{h}=\sum \bar{Y}_{j}(t) \phi_{j}(u),
$$

where $\phi_{j}(u)$ is the standard "hat-function" which takes the value 1 at $u=j h$ and decreases linearly to zero at $u=(j-1) h$ and $u=(j+1) h$. Substituting this form for the solution into the weak formulation (130)-(131) we arrive at the following difference scheme:

$$
\frac{\dot{\mathbf{q}}_{\mathbf{i}-\mathbf{1}}}{6} d_{i}+\frac{\dot{\mathbf{q}}_{\mathbf{i}}}{3}\left(d_{i}+d_{i+1}\right)+\frac{\dot{\mathbf{q}}_{\mathbf{i}+\mathbf{1}}}{6} d_{i+1}=\frac{\mathbf{q}_{\mathbf{i}+\mathbf{1}}-\mathbf{q}_{\mathbf{i}}}{g_{i+1}}-\frac{\mathbf{q}_{\mathbf{i}}-\mathbf{q}_{\mathbf{i}-\mathbf{1}}}{g_{i}}
$$

where

$$
\begin{array}{r}
\mathbf{q}_{\mathbf{i}}=\bar{X}_{i} \mathbf{e}_{\overline{\mathbf{x}}}+\bar{Y}_{i} \mathbf{e}_{\overline{\mathbf{y}}}, \\
g_{i}=\left|\mathbf{q}_{\mathbf{i}}-\mathbf{q}_{\mathbf{i}-\mathbf{1}}\right|,
\end{array}
$$

and

$$
d_{i}=\left(b\left(\bar{X}_{i}-\bar{X}_{i-1}\right)^{2}+m_{1}\left(\bar{Y}_{i}-\bar{Y}_{i-1}\right)^{2}\right) / g_{i} .
$$

This difference scheme is then solved using a semi-implicit method based on the backward-Euler scheme; taking $g_{i}$ and $d_{i}$ at the old time step but taking $\mathbf{q}_{\mathbf{i}}$ where it appears on the right-hand side at the new time step. Results for the contraction of a vortex ring in a plane at various angles $\theta$ to the $z$-axis are shown in Figure 2.

5.2. Parallel rectilinear vortices. The simplest intervortex interaction that is possible to study is that between two parallel rectilinear vortices. However, even in this simple case, the orientation of the vortices with respect to the $z$-axis will be significant. Consider then a rotation of axes such that the $\bar{z}$-axis makes an angle $\alpha$ with the $z$-axis (see Figure 1), and furthermore let the vortices lie parallel to this 


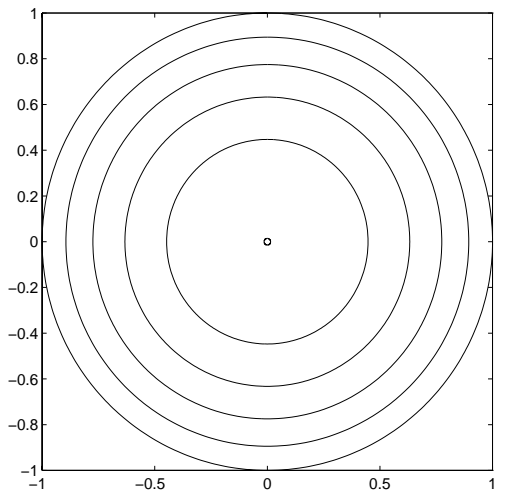

(a)

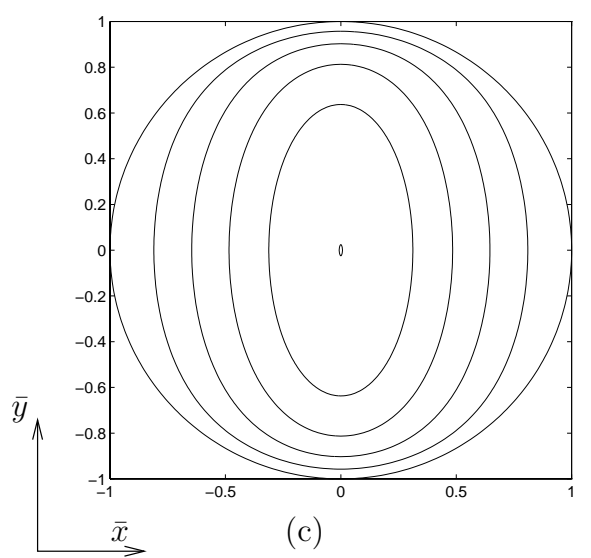

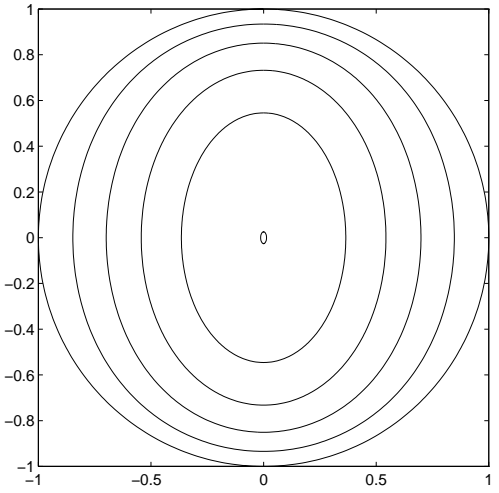

(b)

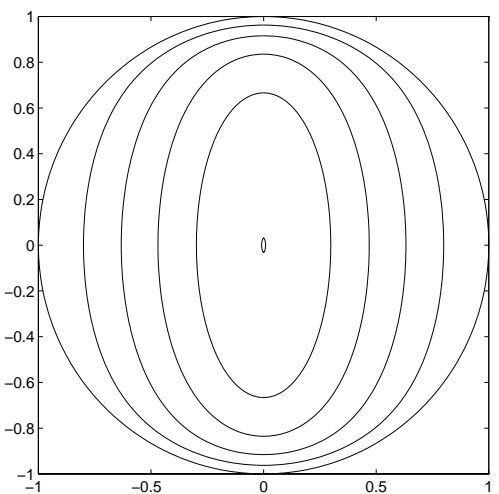

(d)

FIG. 2. The evolution of a vortex ring lying in a plane with normal, making an angle (a) $\theta=0$, (b) $\theta=\pi / 6$, (c) $\theta=\pi / 3$, (d) $\theta=\pi / 2$ with the $z$-axis. The various plots are at equal time intervals and the final plot is at (a) $t=0.250$, (b) $t=0.415$, (c) $t=0.625$, (d) $t=0.708$.

axis. In order to calculate the velocity of one of these vortices one must calculate the magnetic field due to the other and then substitute this into the velocity law at the point of the first vortex (see (125)). In general it suffices to consider the field due to a vortex line lying along the $\bar{z}$-axis. Note that the field takes the form $\mathbf{H}=H_{1}(\bar{x}, \bar{y}) \mathbf{e}_{\overline{\mathbf{x}}}+H_{2}(\bar{x}, \bar{y}) \mathbf{e}_{\overline{\mathbf{y}}}+H_{3}(\bar{x}, \bar{y}) \mathbf{e}_{\overline{\mathbf{z}}}$ and thus that the first two components may be written in terms of a stream function

$$
H_{1}=-\frac{\partial \psi}{\partial y}, \quad H_{2}=\frac{\partial \psi}{\partial x},
$$

where the bars have now been dropped. Substitution of the above form for the magnetic field into (127) then yields the following coupled elliptic equations for $\mathrm{H}_{3}$ and $\psi$ :

$$
\begin{array}{r}
\frac{H_{3 y y}}{m_{1} d}+m_{1} H_{3 x x}-H_{3}+\frac{c}{d} \psi_{y}=-2 \pi \delta(x) \delta(y), \\
\psi_{x x}+\psi_{y y}-\frac{\psi}{d}+\frac{c}{d} H_{3 y}=0,
\end{array}
$$


where the parameters $b, c$, and $d$ are functions of the anisotropy and the angle between the $\bar{z}$-axis and the $z$-axis:

$$
\begin{gathered}
b=m_{1} \cos ^{2} \alpha+\frac{1}{m_{1}^{2}} \sin ^{2} \alpha, \\
c=\cos \alpha \sin \alpha\left(m_{1}-\frac{1}{m_{1}^{2}}\right), \\
d=m_{1} \sin ^{2} \alpha+\frac{1}{m_{1}^{2}} \cos ^{2} \alpha .
\end{gathered}
$$

In the absence of boundaries it is clear that the magnetic field should decay as $x^{2}+$ $y^{2} \rightarrow \infty$, and so we impose the boundary data

$$
H_{3} \rightarrow 0 \quad \text { and } \quad \psi \rightarrow 0 \quad \text { as } \quad x^{2}+y^{2} \rightarrow \infty
$$

The velocity of another similarly aligned vortex parallel to the $\bar{z}$-axis but at $(x, y)=$ $\left(q_{1}, q_{2}\right)$ is calculated from (125) and found to be, after scaling time with $\beta / 2$,

$$
\mathbf{v}=-\left.\left(m_{1} d^{1 / 2} H_{3 x} \mathbf{e}_{\mathbf{x}}+\frac{1}{m_{1} d^{1 / 2}} H_{3 y} \mathbf{e}_{\mathbf{y}}\right)\right|_{(x, y)=\left(q_{1}, q_{2}\right)} .
$$

We now proceed to calculate the velocity field of a vortex.

Exact solutions. Exact solutions to (135) and (136) can easily be obtained only when $c=0$ and these equations decouple, that is, in the cases $\alpha=0$ and $\alpha=\pi / 2$. When $\alpha=0$ the solution is almost identical to that of the isotropic vortex. In fact we find

$$
\begin{gathered}
H_{3}=\frac{1}{m_{1}} K_{0}\left(\sqrt{\frac{x^{2}+y^{2}}{m_{1}}}\right), \quad \psi=0, \\
\mathbf{v}=m_{1}^{-3 / 2} \frac{x}{\sqrt{x^{2}+y^{2}}} K_{1}\left(\sqrt{\frac{x^{2}+y^{2}}{m_{1}}}\right) \mathbf{e}_{\mathbf{x}}+m_{1}^{-3 / 2} \frac{y}{\sqrt{x^{2}+y^{2}}} K_{1}\left(\sqrt{\frac{x^{2}+y^{2}}{m_{1}}}\right) \mathbf{e}_{\mathbf{y}},
\end{gathered}
$$

whereas in the case $\alpha=\pi / 2$ we find

$$
\begin{aligned}
H_{3}= & m_{1}^{1 / 2} K_{0}\left(\sqrt{x^{2} / m_{1}+m_{1}^{2} y^{2}}\right), \quad \psi=0, \\
\mathbf{v}= & \frac{m_{1} x}{\sqrt{x^{2} / m_{1}+m_{1}^{2} y^{2}}} K_{1}\left(\sqrt{x^{2} / m_{1}+m_{1}^{2} y^{2}}\right) \mathbf{e}_{\mathbf{x}}, \\
& +\frac{m_{1} y}{\sqrt{x^{2} / m_{1}+m_{1}^{2} y^{2}}} K_{1}\left(\sqrt{x^{2} / m_{1}+m_{1}^{2} y^{2}}\right) \mathbf{e}_{\mathbf{y}} .
\end{aligned}
$$

Numerical solution. Numerical solution of equations (135) and (136) is most easily made using a finite element method, since writing (135) in weak form means that only a convolution of the distribution, on the right-hand side of this equation, with a test function need be considered. Furthermore as the two equations are relatively weakly coupled we chose to consider them independently, taking the derivative of $\psi$ where it occurs in (135) and the derivative of $H_{3}$ where it occurs in (136) to the right-hand side of these equations. We then use a successive overrelaxation method to solve the 


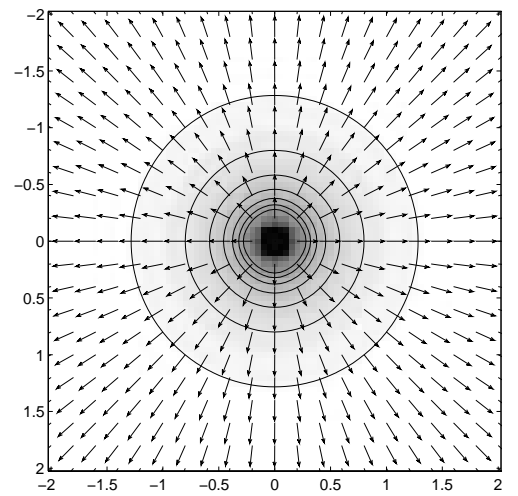

(a)

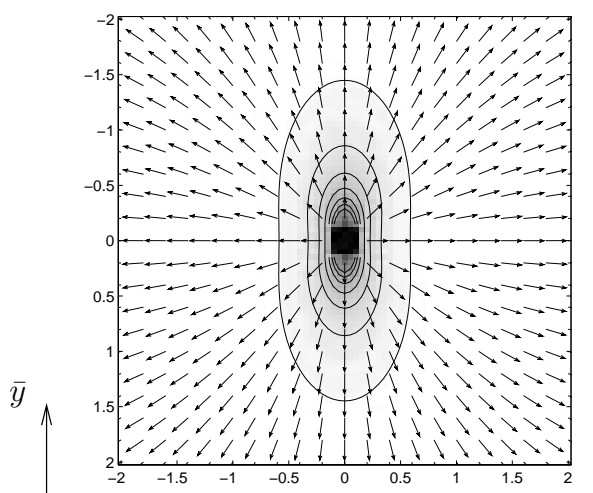

(c)

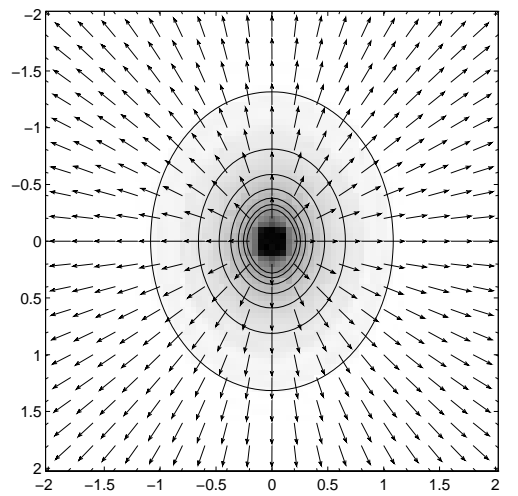

(b)

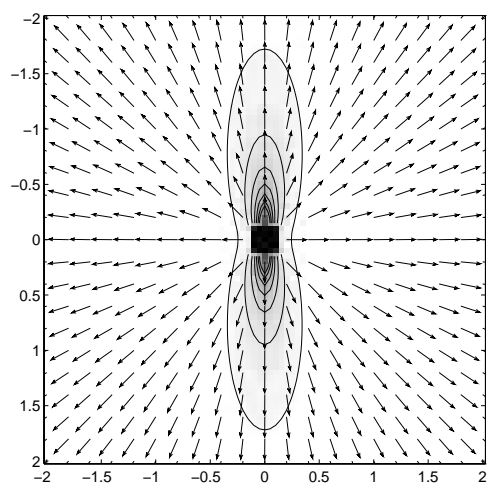

(d)

$\bar{x}$

FIG. 3. The velocity field induced by a positive vortex lying along the $z^{\prime}$-axis, where the $z^{\prime}$-axis makes an angle (a) $\alpha=0$, (b) $\alpha=\pi / 6$, (c) $\alpha=\pi / 3$, (d) $\alpha=\pi / 2$ with the $z$-axis. The arrows show the direction of the velocity while the contour plot is of the magnitude of the velocity with contours at $0,2,4,6,8,10,12,14$. Darker regions indicate larger velocities.

resulting finite difference scheme, updating the values of $\psi$ and $H_{3}$ where they appear on the right-hand side of the finite difference version of these equations after each step of the iteration with the new values. Although there is no guarantee that this procedure will converge, the calculation of a residue, enables us to determine if it is indeed doing so, and in fact we could find no case where it did not.

The results of these calculations for the particular case $m_{1}=1 / 2$ and various angles $\alpha$ are displayed in Figure 3, in which a plot of the direction of the velocity field and its magnitude are made. We simulated the boundary conditions (137) by imposing zero boundary data on the edge of a square, with sides of length 10 and the vortex at its middle.

6. Conclusion. We have considered the behavior of a system of vortices in a high- $T_{c}$ superconductor described by anisotropic Ginzburg-Landau equations. We began by analyzing a system of well-separated vortices and were able to derive a law of motion analogous to the local induction approximation, in which the vortex moves in 
the normal direction with a speed proportional to its curvature, where the constant of proportionality depends upon the angle the tangent to the vortex line makes with the $c$-axis. We then considered a system of densely packed vortices and were able to derive a mean-field model, in which the individual vortices are replaced by a vortex density. These results generalized the recent work in [4] and [6] to anisotropic superconductors. Next we looked at a system of sparsely packed vortices with sufficiently small curvature such that their motion is principally externally driven, a particular important example of this scenario being a group of parallel rectilinear vortices. Finally we looked at examples of the self-induced motion of a planar vortex loop and of the velocity field induced by a rectilinear vortex at various angles to the axes of anisotropy. We found an exact solution to the law of motion given by the local induction approximation corresponding to an elliptical vortex shrinking to a point in finite time. Numerical solutions of initially circular vortices showed the vortex to become elongated, with the portions of the vortex closest to being aligned with the $z$-axis (the axis of principal anisotropy) moving more slowly. When we examined the velocity of repulsion of a pair of rectilinear vortices aligned at an angle to the $z$-axis, we found that the effects of the anisotropy were felt most strongly when the vortices were perpendicular to this axis. In this case the vortices repel most strongly if the line of centers is also perpendicular to the $z$-axis (so that they lie in the $x y$-plane) and only weakly if the line of centers is along the $z$-axis (so that the vortices lie in the $x z$-plane).

\section{REFERENCES}

[1] G.K. BAtchelor, An Introduction to Fluid Dynamics, Cambridge University Press, London, 1967.

[2] G. Blatter, V.B. Geshkenbein, And A.I. Larkin, From isotropic to anisotropic supercondutors: A scaling approach. Phys. Lett., 68 (1992), p. 875.

[3] S.J. Chapman, S.D. Howison, And J.R. OCKenden, Macroscopic models for superconductivity, SIAM Rev., 34 (1992), pp. 529-560.

[4] S.J. Chapman, A mean-field model of superconducting vortices in three dimensions, SIAM J. Appl. Math., 55 (1995), pp. 1259-1274.

[5] S.J. Chapman, Q. Du, And M.D. Gunzburger, On the Lawrence-Doniach and anisotropic Ginzburg-Landau models for layered superconductors, SIAM J. Appl. Math., 55 (1995), pp. 156-174.

[6] S.J. Chapman and G. Richardson, Motion of vortices in type-II superconductors, SIAM J. Appl. Math., 55 (1995), pp. 1275-1296.

[7] X. Chen, C.M. Elliott, AND Q. TANG, Shooting method for vortex solutions of a complexvalued Ginzburg-Landau equation, Proc. Roy. Soc. Edinburgh Sect. A, 124 (1994), p. 1075.

[8] Q. Du, M.D. Gunzburger, And J.S. Peterson, Analysis and approximation of the GinzburgLandau model of superconductivity, SIAM Rev., 34 (1992), p. 54.

[9] G. DzIuk, Convergence of a semi-discrete scheme for the curve shortening flow, Math. Models Methods Appl. Sci., 4 (1994), p. 589.

[10] V.M. Genkin And A.S. MeL'Nikov, Motion of Abrikosov vortices in anisotropic superconductors, Zh. Eksp. Teor. Fiz., 95 (1989), p. 2170.

[11] V.L. Ginzburg And L.D. Landau, On the theory of superconductivity. J.E.T.P., 20 (1950), p. 1064.

[12] L.P. Gor'Kov And G.M. Eliashburg, Generalisation of the Ginzburg-Landau equations for non-stationary problems in the case of alloys with paramagnetic impurities, Sov. Phys. J.E.T.P., 27 (1968), p. 328.

[13] L.P. GOR'KOV AND N.B. KopNIN, Viscous vortex flow in superconductors with paramagnetic impurities, Sov. Phys. J.E.T.P., 33 (1971), p. 1251.

[14] R.A. Klemm And J.R. Clem, Lower critical field of an anisotropic type-II superconductor, Phys. Rev. B, 21 (1980), p. 1868.

[15] W. Lawrence and S. Doniach, Theory of layer structure superconductors, Proc. 12th Internat. Conf. on Low Temp. Phys., Academic Press of Japan, Kyoto, pp. 361-362, 1971.

[16] P.G. Saffman, Vortex Dynamics, Cambridge University Press, London, 1992.

[17] M. Van Dyke, Perturbation Methods in Fluid Dynamics, Parabolic Press, Stanford, 1975. 